\title{
Improving accessibility of trust guidelines and protocols at the Great Western Hospital, Swindon
}

Ian Robertson, Abigail Smith, Jennifer Tucker, Erica Cilia, Kangni Chen, Rose Marion, Julian Nesbitt, Steve Ramcharitar, Mala Cathiavadi Greamspet

Great Western Hospital, Swindon

\begin{abstract}
Trust guidelines and policies outline recommendations for the management of common clinical and non-clinical situations, serving to standardise best practice. Prior to this project, there was no consolidated location for these documents. Lack of organisational structure and inadequate search functionality within the trust intranet led to time wasted locating information, acting outside of recognised best practice, and ultimately potentially compromising patient safety.
\end{abstract}

We surveyed 55 junior doctors, $95 \%$ of respondents were dependent on guidelines on a daily basis. $20 \%$ spending greater than 5 minutes to locate protocols and $38 \%$ unable to locate some relevant documents at all. We analysed the time taken for junior doctors to locate six randomly selected protocols. Pre-intervention mean time was 133 seconds (on six occasions doctors were unable to locate the guideline).

All trust guidelines and protocols currently available on the intranet were collated, consolidated, and renamed according to content. These were then re-alphabetised and new search terms linked to each document. Existing links were then uploaded and a single web page made available via the trust intranet homepage. The new page was publicised by email, posters and interdepartmental presentations.

In our post intervention survey, $97 \%$ of respondents were aware of the project and had made use of the page. All protocols were located during re-testing with $90 \%$ of those resurveyed stating it was easier to locate protocols. Overall, a reduction in the time and number of clicks required to locate protocols was demonstrated: mean time 16 seconds vs 133 seconds pre-intervention $(n=60) .53 \%$ of guidelines located in $<30$ s and $86 \%<2$ minutes.

\section{Problem}

Trust guidelines and policies are documents outlining recommendations for the management of important clinical and nonclinical situations. Such documents serve to standardise best practice and should be easily accessible to all staff members. At the Great Western Hospital in Swindon, content of these documents is maintained, updated and regulated by the Trust. Documents are then hosted on the intranet, where they are made available to clinicians. Staff of all levels depend on the availability of this information to make clinical decisions on a daily basis.

Prior to this project there was no Trustwide consolidated location for these documents. Lack of organisational structure and inadequate search functionality led to time wasted locating information, acting outside of recognised best practice, and ultimately potentially compromised patient safety.

\section{Background}

Much interdepartmental variation exists in the methods used to organise and disseminate trust guidelines within NHS hospitals. This leads to duplicated effort on the part of the provider and frustration among users. Carthey et al highlight the often incongruous nature of the availability of centralised information and the confusion created by obscure naming of documents.[1] They cite the use of specific "drives" departmental servers without Trustwide access.

Feder et al suggest investigating barriers to care when designing solutions to problems disseminating information to their workforce.[2] Individual Trusts face specific challenges, so the opinions of users are pivotal to designing any innovative framework for guidelines.

Accessibility to Trust guidelines is not a problem unique to Great Western Hospital, Swindon. A similar project was undertaken by junior doctors in North Bristol in 2012, "Improving the accessibility of Trust guidelines for junior doctors at North Bristol NHS Trust".[3] Links to guidelines via the Trust intranet were re-designed to allow access via the homepage. A significant reduction in time taken to locate guidelines was demonstrated when using a novel single point of access.

\section{Baseline measurement}

Our first objective was to survey staff opinions regarding the accessibility of Trust guidelines and evaluate the time taken to locate these documents. In November 2013 we conducted an online survey of doctors of all levels currently employed by the Trust. We had 55 responses from a range of departments and 
seniority levels, but from primarily junior doctors. Of this cohort, 95\% (53) were dependent on protocols and guidelines for clinical decision making, 89\% (47) accessed Trust guidelines or protocols at least weekly, with most needing daily access. Of note, $81 \%$ (42) felt than an inability to promptly locate protocols had direct impact on clinical duties.

Respondents were asked to identity their most recently searched guideline and approximate the time taken. Only 20\% (11) reported a search time of less than 2 mins, 22\% (12) between 2 and 5 mins and $20 \%$ (11) more than 5 mins. Of concern, $38 \%$ (21) of respondents were unable to locate any document whatsoever. In addition we offered respondents the opportunity to input free text comments. Some examples included:

"Very frustrating - particularly when l've worked in a previous hospital where you can find protocols within seconds."

"I would estimate I lose at least 15 minutes every single shift looking for protocols."

"It's a patient safety concern if we can't find protocols."

Some respondents reported they only knew how to locate a guideline if they had spent time searching for it in the past. This is particularly relevant given that junior doctors often rotate through a range of departments during their time in the Trust, spending only a few months in each post.

Initial survey results suggested that there was great potential for improvement. $60 \%$ felt that an increased availability of Trust guidelines would significantly improve their ability to work effectively and the remaining $40 \%$ felt that it would improve their ability to work effectively to some degree. We asked respondents how they felt guidelines would be best made available to doctors. $71 \%$ suggested a separate location on the intranet. Only one respondent felt that the way things were at the moment was acceptable.

To supplement subjective opinions with objective measures, we randomly selected six protocols and invited a representative sample of five junior doctors to locate each document while recording the time elapsed. Protocols included: refeeding syndrome, massive haemorrhage, insulin sliding scale, diabetic ketoacidosis, acute kidney injury, and IV heparin. Once the participants opened the intranet homepage, the timer was started and then stopped when the correct document was successfully opened. Overall 30 searches were performed, of these $80 \%$ (24 searches) successfully located the protocol and $20 \%$ (six) were unable and abandoned the search. An upper time limit was not set, but rather the doctor was allowed to stop searching when they felt they would ordinarily abandon the search in their day to day practice. The time to complete the search ranged from 9 seconds to 9 minutes.

Where the protocols were successfully found the averages times taken are illustrated in figure 1.

Participants were frequently unable to locate the insulin sliding scale and acute kidney injury protocols. This is particularly concerning considering the wide range of specialties and departments to which these guidelines are relevant. While some protocols were found relatively quickly (for example diabetic ketoacidosis with an average of 26.4 seconds) we speculated that even these timings could be significantly improved if all protocols were to be found in a single location.

See supplementary file: ds3928.jpg - "Fig. 1"

\section{Design}

Our primary intervention was to design and implement a single intranet webpage to collate and host Trust guidelines and policy documents. We aimed to do this by collecting all current guidelines available on the intranet, simplifying the nomenclature as necessary, and then assigning appropriate search terms to each document. We considered various options for categorising links to documents. Ideas included by department or speciality, but given that many protocols are used across multiple specialities (for example acute kidney injury) we decided they would be best indexed in an alphabetical list. This also meant that the search function within the webpage could be more effectively utilised. This allows for documents to be located under different search terms for example, chest pain guidelines listed with keywords: chest pain, myocardial infarction, MI, acute coronary syndrome, ACS.

We wanted to create a single webpage that was easily accessible via the "useful links" list on the hospital's intranet homepage. This means that it will not only be quick for clinical staff to find, but that the guidelines will also be in an obvious location for for those unfamiliar with the Trust intranet. We liased closely with the Trust IT services in order to create a new webpage on which to host the guidelines and subsequently link this to the Trust homepage.

In order to maintain current versions and reduce frustration and confusion for clinical staff, historical links (accessible via a range of sites within the intranet) will continue to be active and our webpage acts as a shortcut to these. These links will be automatically updated when guidelines are updated by a department in the future, giving sustainability to the project.

Additionally, the page can be edited or augmented as necessary via the Umbraco content management software. Prior to leaving the Trust, the project team members will hand over the management and maintenance of the site to a guidelines committee who will be responsible for uploading and maintaining new clinical guidelines. We anticipated that the entire project would take around three months to design and implement. We successfully launched the page in March 2014.

\section{Strategy}

From the outset we had a clear idea of our end goal but this process was broken down into two 'Plan-Do-Study-Act' (PDSA) cycles (see figure 3). This made the project more manageable and gave us the chance to reflect and make changes after our initial interventions. 
BMJ Quality Improvement Reports

The first PDSA cycle involved creating a webpage to host guidelines and protocols. This was initially accessible via the emergency department intranet page (figure 4) because we were able to gain permission to use this as a host location. We planned the intervention as a group and divided tasks between us such as locating protocols, determining search terms, creating the webpage and testing the links. Informal feedback was sought from colleagues who held a general consensus that whilst the page itself was helpful as it was a single location for protocols, it still took too long to navigate to the page itself (see figure 5 for "number of clicks").

The second PDSA cycle focused on making navigation to the webpage quicker and easier. This required further discussions with the IT department who agreed to trial hosting our webpage on a direct link from the intranet homepage. This was done by creating a shortcut link entitled "clinical guidelines A-Z" on the "useful links' area of the intranet homepage.

We resurveyed junior doctors using an online survey tool and objectively measured the time taken to locate the documents in their new location. We received significant positive feedback about the page itself and the ease with which it could be found. Figure 3 demonstrates two PDSA cycles and figure 5 shows the reduction in "number of clicks" required to reach a protocol after each cycle was complete.

The only negative feedback received following this second cycle related to protocols that were not yet hosted on the webpage. This identified protocols that we had initially been unable to locate or that had been subsequently created since we made the webpage. We were able to overcome this challenge by uploading the documents ourselves.

See supplementary file: ds4329.pdf - "Fig 34 5"

\section{Post-measurement}

\section{Number of clicks}

It was noted that the one of the main reasons many of the documents were difficult and time consuming to locate was due to the number of menu options and links staff had to navigate. For example, reaching the chest pain protocol pre-intervention took six steps. Post-intervention, this was reduced to three steps for all documents. The average number of clicks to reach individual documents was reduced by $53 \%$ (figure 5 ).

\section{Timings}

Following implementation of the webpage we repeated our methodology for time objective measurements. We asked a representative sample of five junior doctors to locate the same documents hosted on the Trust intranet. Pre-intervention mean time was 133 seconds (on six occasions doctors were unable to locate the guideline). Post-intervention, all guidelines were located and times were reduced in all cases. Mean time to successfully locate the document was 16 seconds $(n=30)$. Using a student T-test this result was highly statistically significant $(p<0.005)$ (see figure 6 ).
See supplementary file: ds4330.pdf - "Fig 6"

\section{Lessons and limitations}

This project has taught us the importance of team working to achieve a common goal. Most importantly, we have learnt that simple interventions have the potential to make a significant difference not only to staff, but also the Trust and most importantly patient safety.

During this project we faced many challenges, only some of which we had anticipated. Locating over 300 documents hosted across more than 50 different pages proved labourious. We achieved this by dividing into teams to tackle different specialties. Once each team had exhaustively collected all documents, we then consolidated them into a database to produce a master document.

We initially struggled to demonstrate the clinical relevance of the project to the IT department. It is sometimes difficult for non-clinical staff to appreciate the importance of timely access to clinical information. We used survey analysis and objective data to demonstrate the frustrations of junior doctors and the potential time savings, revealing the importance of partnership with IT.

Guidelines hosted on the intranet are updated on a regular basis in line with current best evidence. The microbiology department expressed concerns that a static link to the antibiotic guidelines could introduce the risk of clinical staff accessing outdated information. We acknowledged these concerns and ensured that any links to regularly updated content referred users to the most current version, allowing individual specialties the flexibility to upload the latest evidence and provide dynamic content.

We noted that search functionality was ineffective prior to the development of the new consolidated page and implemented a robust solution of keywords attached to each hyperlink allowing users to search within the page. Work to implement a more elegant search solution is ongoing.

Future challenges of this project will be the continual maintenance of the content as new guidelines are added to the intranet. It is important to note that updating the content of individual guidelines is beyond the scope of this project, although work is currently underway to review the content of each guideline in accordance with best practice.

At present the webpage is only accessible via the Trust intranet using Trust based computers. We hope that in the future a smartphone app could be developed to allow doctors on-the-go access to important information.

\section{Conclusion}

From the outset it was noted by clinical staff that locating guidelines, which we depend upon on a daily basis, was difficult and time consuming. It becomes the responsibility of those most affected to take the initiative and make a positive change. In a world 


\section{BMJ Quality Improvement Reports}

of Google and Apple's Siri where search results are lightening fast and impressively intuitive, we have come to expect the same of hospital IT systems.

Our team have worked hard to collect convincing evidence of the inadequacies of the current system, and to demonstrate objectively the time wasted spent lost in a sea of hyperlinks. We sought the opinions of other clinical staff and incorporated their views into the development of a solution and produced a single unified page to host all the documents in one place.

It is testament to the dedication of the team that we demonstrated such dramatically improved objective measures and positive feedback. We hope that this project serves to save time and improve patient safety, all at minimal cost. The essence of this project was elegantly encapsulated by one anonymous comment: "Well done for setting this up - long overdue and a victory for common sense!"

\section{References}

1. ?Carthey J, Walker S, Deelchand V. Breaking the rules: understanding non-compliance with policies and guidelines. BMJ 2011;343:d5283.

2. ?Gene Feder MERGCGJG. Clinical guidelines: Using clinical guidelines. BMJ 1999 Mar 13;318(7185):728.

3. Tarrant M, Honeyman C, Aquilina A, Young K. 'Improving the accessibility of trust guidelines for junior doctors at North Bristol NHS trust'. BMJ Quality Improvement Rep 2014; u202211.w1101 doi: 10.1136/bmjqualiy.u202211.w1101.

\section{Declaration of interests}

Nothing to declare.

\section{Acknowledgements}

Abigail Smith and lan Robertson are the joint first authors of this project.

Many thanks to our supervisor Dr Deepak Gupta, Trisha Reeves and Luke Newman from the GWH Academy for supporting the QI team, Dr. Mala Greamspet, the head of the QI team Dr. Steve Ramcharitar, and the rest of the QI team. Also thanks to Luke Newman QI project administrator and the rest of the education department at Great Western Hospital, Swindon for their help and support on this project. 